\title{
BMJ Open Incidence of omental metastasis in uterine serous carcinoma: study protocol for a systematic review and meta-analysis
}

\author{
Cui Hu, ${ }^{1,2}$ Lin-Lin Zhang, ${ }^{1}$ Yu Cheng, ${ }^{1}$ Fei-Xue Xue, ${ }^{1}$ Ya Jia, ${ }^{3}$ Qin Zhao-Juan, ${ }^{3}$ \\ Du Yi, ${ }^{3}$ Zhang Qian-Wen, ${ }^{3}$ He Yue-Dong, ${ }^{3}$ Zheng $\mathrm{Ai}^{3}{ }^{3} \mathrm{Yu} \mathrm{Xu}$ (i) ${ }^{3}$
}

To cite: Hu C, Zhang L-L, Cheng Y, et al. Incidence of omental metastasis in uterine serous carcinoma: study protocol for a systematic review and meta-analysis. BMJ Open 2021;11:e043141. doi:10.1136/ bmjopen-2020-043141

- Prepublication history and additional material for this paper are available online. To view these files, please visit the journal online (http://dx.doi. org/10.1136/bmjopen-2020043141).

Received 27 July 2020

Revised 19 December 2020

Accepted 06 January 2021

D Check for updates

C Author(s) (or their employer(s)) 2021. Re-use permitted under CC BY-NC. No commercial re-use. See rights and permissions. Published by BMJ.

${ }^{1}$ Department of Obstetrics and Gynaecology, Mianzhu People's Hospital, Mianzhu, China

${ }^{2}$ Department of Obstetrics and Gynaecology, Southwest Medical University, Luzhou, Sichuan,

China

${ }^{3}$ Department of Obstetrics and Gynaecology, Sichuan University West China Second University Hospital, Chengdu, Sichuan, China

Correspondence to

Dr Yu Xu; xuyu@stu.scu.edu.cn

\section{ABSTRACT}

Introduction Uterine serous carcinoma accounts for only about $10 \%$ of all endometrial cancers but this subtype is the most common amongst non-endometrioid endometrium cancers and contributes to more than half of recurrence and deaths attributed to endometrial cancers. A more extensive surgical staging and adjuvant therapies for uterine serous carcinoma are recommended by many guidelines. However, guidelines vary on recommendations for the methods that should be used for omentum assessment in uterine serous carcinoma and the previously reported incidence of omental metastasis in uterine serous carcinoma had a wide range because of the heterogeneity among these studies. As far as we know, there are no systematic review and meta-analysis available on this topic. The aim of our proposed study is to statistically synthesise the data examining the incidence of omental metastasis in uterine serous carcinoma.

Methods and analysis Systematic searches of three databases (PubMed, Embase and Web of Science) will be performed using prespecified search strategies. We will include original studies that reported incidence of omental metastasis in uterine serous carcinoma and are published before 30 August 2020. Our different investigators will independently conduct the eligible study selection, assess the quality of included studies and extract the needed data If appropriate, the relevant data will be pooled through a random-effect or fixed-effect meta-analysis based on the heterogeneity among included studies. We will evaluate the overall quality of evidence using appropriate methods. Ethics and dissemination This proposed study will be based on published data, and thus, there is no requirement for ethics approval. We aim to publish the results of this study in a peer-reviewed journal with good visibility for the fields of gynaecology and gynecologic oncology. PROSPERO registration number CRD42020200891.

\section{INTRODUCTION}

According to the revised 2014 World Health Organization Classification of Tumours of the Female Reproductive Organs, ${ }^{1}$ endometrial cancer is divided into the following histological subtypes: endometrioid adenocarcinoma, mucous carcinoma, serous carcinoma, clear cell carcinoma, carcinosarcoma and so on. Uterine serous carcinoma (USC) was first reported by Hendrickson et al in

\section{Strengths and limitations of this study}

- This will be the first systematic review and metaanalysis to explore the incidence of omental metastasis in uterine serous carcinoma.

- We will conduct our research in strict accordance with the relevant methodological guidelines of systematic review and meta-analysis.

- A limitation of our study is that only the articles published in English will be included because of the limited resource and the authors' linguistic competence.

- Original studies may have small sample size because of the rarity of uterine serous carcinoma; majority of them may be retrospectively designed with some degree of recall bias, which may compromise the results of our study.

- Considering the possibility of high heterogeneity among the eligible studies, the meta-analysis may will not to be performed.

1982 as a distinct subtype of endometrial carcinoma and had many histological similarities with ovarian serous carcinoma. ${ }^{2-4}$ USC makes up only about $10 \%$ of all endometrial cancers ${ }^{5}$ but is the most common subtype of non-endometrioid cancer and contributes to more than $50 \%$ of recurrence and the total number of deaths attributed to endometrial carcinoma. $^{2}{ }^{6-9}$ When compared with welldifferentiated endometrioid cancer, USC is more commonly found in the elderly and has been characterised by a propensity for spread to the upper abdomen, high recurrence risk and a worse prognosis. ${ }^{810-13}$

So far, the patterns of cancer spread are not well understood in USC. Indeed, some studies have demonstrated that metastatic disease can occur even in the absence of myometrial invasion and lymphovascular spread. ${ }^{814}$ Given that USC has more advanced stages at the time of presentation, high risk of recurrence and relatively poor prognosis, a more extensive staging surgery including total hysterectomy, bilateral salpingo-oophorectomy, 
omental assessment, peritoneal biopsies and pelvic and para-aortic lymph node assessment is recommended by many clinical practice guidelines. ${ }^{515-18}$ But, the methods of omental assessment for USC vary from guideline to guideline. For example, omental biopsy not omentectomy is recommended for the surgical treatment of USC by the National Comprehensive Cancer Network (NCCN), while omentectomy is mandatory for the surgical staging of USC according to the ESMO-ESGO-ESTRO consensus. ${ }^{515}$

However, even in patients with non-invasive serous carcinoma limited to the endometrium who undergone extensive staging surgery, up to $30 \%$ of women developed disease recurrence, ${ }^{19}$ questioning the significance of extensive staging surgery as well as suggesting the value of postoperative adjuvant therapy for USC. Moreover, Gehrig $e t a l^{20}$ conducted a study to compare the pathological and visual assessment of the omentum in USC and found that there was no case where the stage was upstaged by a pathological finding of occult disease to the omentum. Hence, the role of routine omentectomy for USC remains controversial. However, there has been a lot of studies that explored the incidence of omental metastasis in USC, but the results varied within a wide range because of the different sample size, study design and study setting. ${ }^{814} 20-25$

To our knowledge, no systematic review and metaanalysis have been conducted on this topic. Our proposed systematic review and meta-analysis aim to provide relatively accurate incidence of omental metastasis in USC by pooled analyses of published data and to inform researchers and clinicians about the practical significance of routine omentectomy for USC.

\section{STUDY POPULATION}

Patients with a histopathological diagnosis of primary USC.

\section{OUTCOMES}

1. The overall incidence of omental metastasis in USC.

2. The incidence of gross omental metastasis in USC.

3. The incidence of occult omental metastasis in USC.

4. The incidence of omental metastasis in clinical stage I USC.

\section{REVIEW QUESTION}

What is the incidence of omental metastasis in USC?

\section{METHODS}

\section{Standards}

This proposed systematic review and meta-analysis will be conducted in accordance with the standard guidelines of 'Meta-analysis Of Observational Studies in Epidemi$\operatorname{ology}^{26 \text { ' }}$ and 'Preferred Reporting Items for Systematic Reviews and Meta-Analyses ${ }^{27}$. This protocol manuscript follows the Preferred Reporting Items for Systematic
Reviews and Meta-Analyses Protocols (PRISMA-P) guidelines. ${ }^{28}$ See online supplemental material 1 for the PRISMA-P checklist.

\section{Search strategy}

In this proposed systematic review and meta-analysis, we will perform systematic literature searches to identify qualifying studies published from database inception until 30 August 2020, in the following there electronic databases: PubMed, Embase and Web of Science. We will restrict searches in articles published in English. To make literature search as comprehensive as possible, we will select keywords informed by medical subject headings as follows: 'non-endometrioid endometrial neoplasm', 'non-endometrioid endometrial carcinoma', 'nonendometrioid endometrial cancer', 'non-endometrioid endometrium cancer', 'non-endometrioid endometrium carcinoma', 'non-endometrioid endometrium neoplasm', 'non-ECC', 'uterine serous carcinoma', 'uterine serous cancer', 'uterine serous neoplasm', 'serous endometrial carcinoma', 'serous endometrial cancer', 'serous endometrial neoplasm', 'serous endometrium cancer', 'serous endometrium carcinoma', 'serous endometrium neoplasm', 'serous carcinoma of the endometrium', 'serous cancer of the endometrium', 'uterine papillary serous carcinoma' or 'UPSC' for disease; 'omentectomy', 'omental biopsy', 'surgical staging', 'surgical treatment' or 'comprehensive surgery' for intervention; and 'omental metastasis', 'omental metastases' or 'extrauterine spread' for the outcome. The search terms will be combined using Boolean logic (AND, OR) where needed. The precise search strategies for one of the databases (PubMed) can be found in the online supplemental material 2. We will also manually check the reference list of the included studies to find any other eligible studies that will not be available in the electronic databases. The literature search will be performed by three members of our research team (YX, YJ and Z-JQ). To make a highquality literature search, the search will be rechecked by an epidemiologist (Y-DH).

\section{Study selection}

Retrieved records from the database and hand searches will be entered into the EndNote reference manager (V.X9), and duplicates will be removed. Titles and abstracts of the remaining records will be screened for relevance by there reviewers (YX, YJ and Z-JQ) who were blind to each other's activities. Irrelevant titles and abstracts will be eliminated. Potentially eligible full-text papers will be obtained and inspected to determine eligibility by the same independent reviewers. For full texts that will not be obtained, an attempt to request a copy from the authors directly will be made.

We predefined the inclusion criteria for eligible studies as follows: (1) original studies published until 30 August 2020; (2) English-language articles whether they had been published in scientific meetings or in peer-reviewed journals; (3) the study population was patients with USC; (4) 
abstracts that had the incidence of omental metastasis in USC; (5) studies reported incidence data and incidence rates separately or when they provided sufficient data to allow calculations, any type of study design was accepted, whether it was randomised controlled or observational, prospective or retrospective; and (6) the International Federation of Gynaecology and Obstetrics staging system was used for diagnosis.

Articles were excluded for the following reasons: (1) they were case reports, series case report that the number of cases is fewer than twenty, review articles, protocols, short communications, personal opinions, practice guidelines, commentaries, notes, editorials, letters or laboratory research; (2) they did not report sufficient data and failed to contact the authors for relevant information; and (3) if more than one study evaluated the same population, the smaller one was excluded.

\section{Data extraction}

We will design, pilot and modify a study-specific table before data extraction. Four members of the review team (YX, YJ, Z-JQ and YD) will independently extract the following data of the eligible studies: name of the first author, year of publication, country, age of samples, design and period of each study, preoperative stage, surgical-pathological stage, type of omental evaluation (omentectomy, infracolic omentectomy or omental biopsy), number of patients who had an omental assessment, number of patients who had omental metastasis, number of patients who had occult omental metastasis and number of patients who had gross omental metastasis. To make sure of the integrity of the data gathered, we will cross-check the results of data extraction; we will discuss it with each other when a disagreement arises. When multiple articles reporting data from the same study population happens, the most comprehensive and accurate data will be used. In cases where the articles reported on different timeframes or subgroups (stage), all non-overlapping data will be included. In the cases of relevant papers in which the required data were not reported, the corresponding authors of these studies will be contacted by email to obtain any information needed relating to effect estimates.

\section{Quality appraisal of included studies}

The methodological quality of eligible studies will be assessed under the guidance of the critical appraisal tool for prevalence studies ${ }^{29}$ with further guidance from Munn et $a l^{30}$ by two independent members (YX and Q-WZ) of our team. According to Joanna Briggs Institute's critical appraisal checklist for studies reporting prevalence data, ${ }^{30}$ there are nine items in total: (1) Was the sample frame appropriate to address the target population? (2) Were study participants sampled in an appropriate way? (3) Was the sample size adequate? (4) Were the study subjects and the setting described in detail? (5) Was the data analysis conducted with sufficient coverage of the identified sample? (6) Were valid methods used for the identification of the condition? (7) Was the condition measured in a standard, reliable way for all participants? (8) Was there an appropriate statistical analysis? (9) Was the response rate adequate, and if not, was the low response rate managed appropriately? Higher total scores indicate better quality and lower risk of a study. Studies will be categorised based on the percentage of yes answers as high quality $(\leq 49 \%)$, moderate quality $(50 \%-$ $69 \%)$ or low quality $(\geq 70 \%)$. For each included study,the overall likelihood of bias will be assessed and reported. Any discrepancies within this procedure will be discussed with Y-DH to achieve consensus.

\section{Data synthesis and assessment of heterogeneity}

For each eligible study, we will calculate or record the incidence rates of omental metastasis based on the event rates. According to the Poisson distribution, we will adopt specific methods to calculate $95 \%$ CIs for incidence rates. ${ }^{31}$ The overall incidence of omental metastasis in USC, the incidence of gross omental metastasis in USC, the incidence of occult omental metastasis in USC and the incidence of omental metastasis in clinical stage I USC will be pooled.

Study heterogeneity will be assessed using the Cochran $\mathrm{Q}$ test and the $\mathrm{I}^{2}$ statistic $^{32}$; the $\mathrm{I}^{2}$ index describes the percentage of total variation across studies due to true heterogeneity rather than chance. ${ }^{33} \mathrm{I}^{2}$ values of $25 \%, 50 \%$ and $75 \%$ are considered to be low, moderate and high degrees of heterogeneity, respectively. ${ }^{32}$ When outcomes presented with low statistical heterogeneity, data will be pooled using a fixed-effect model and corresponding 95\% CIs; with moderate or high statistical heterogeneity, a random-effect model will be adopted.

We will assess the publication bias of included studies by visual inspection of funnel plots and statistical assessment using the Egger test. ${ }^{34}$ The statistical software (Stata, V.15.1/IC, StataCorp LP) will be used for the analyses; two-tailed $\mathrm{p}<0.05$ will be considered statistically significant.

\section{Patient and public involvement}

Patients and/or the public will not be involved in the design, or conduct, or reporting, or dissemination plans of this research.

\section{Current study status}

According to our research plan, the study will not begin until 30 August 2020.

\section{Ethics and dissemination}

This proposed systematic review and meta-analysis will be based on published data, and thus, there is no requirement for ethics approval. To our knowledge, this will be the first systematic review and meta-analysis on the incidence of omental metastasis in USC. Results of this study will aid in the knowledge of the practical significance of omentectomy in the management of USC. We aim to publish the results of this systematic review in a 
peer-reviewed journal with good visibility for the fields of gynaecology and gynaecologic oncology.

\section{DISCUSSION}

USC is a rare subtype of endometrial cancer and accounts for only about $10 \%$ of all cases. ${ }^{5}$ Because of its rarity, there are only a few prospective studies to inform on management strategies; treatment recommendations are generally based on results of small, retrospective single-centre studies, suffered from the inherent nature and bias of retrospective observational study. ${ }^{35}$

Traditionally, extensive surgical treatment, as well as postoperative adjuvant therapy, has been employed for the management of USC due to its aggressive biological behaviour and poor prognosis. ${ }^{35}$ For most patients with USC, surgery is a major part of initial treatment, either comprehensive staging for early-stage disease or debulking, in the case of advanced-stage disease. ${ }^{36}$ The standard surgical staging procedures for USC are total hysterectomy, bilateral salpingo-oophorectomy and lymph node assessment. ${ }^{37}$ In consideration of the tendency USC has to transfer to the peritoneal cavity, many researchers were in favour of omentectomy at the time of comprehensive surgical staging. ${ }^{23}{ }^{38-40}$ However, the standpoints about omentectomy in the management of USC have not been unified. Gehrig et al concluded that routine omental sampling does not need to be included in the surgical staging of USC..$^{20}$ Also, relevant practice guidelines had different recommendations about the methods of omental assessment in the staging surgery for USC. ${ }^{5151618}$ And the reported incidence of omental metastasis in USC varied within a wide range due to the heterogeneity among these studies.

This proposed study has significant clinical importance and will be the first pooled analysis to explore the incidence of omental metastasis in USC. But there are also some possible limitations of our study that we can anticipate. First, the potentially eligible studies may be poorly designed, due to the low incidence of USC; they generally have small samples and were retrospectively single institutional studies. Second, among the included studies, there may have some degree of heterogeneity attributes to different research settings and different assessment methods of omental metastasis. Lastly, it will be that some of the relevant studies fail to provide the data we need and that contacting the authors to obtain the data will not get a response.

Correction notice This article has been corrected since it first published. The provenance and peer review statement has been included.

Contributors $Y X$ and $A Z$ conceived the idea. $Y X, C H, L-L Z, Y C$ and F-XX drafted the protocol. All remaining authors (YJ, Z-JQ, YD, Q-WZ and Y-DH) critically revised all drafts of the manuscript for intellectual content and agreed and approved the final manuscript. $Y X$ is the guarantor of the review.

Funding This study received funding through the Major Research and Development Project of Sichuan Provincial Department of Science and Technology, from the science and technology department of Sichuan Province, Project Grant Number: 2019YFS0402.
Disclaimer The funders' and authors' institutions are not responsible for its content.

Competing interests None declared.

Patient consent for publication Not required.

Provenance and peer review Not commissioned; externally peer reviewed.

Supplemental material This content has been supplied by the author(s). It has not been vetted by BMJ Publishing Group Limited (BMJ) and may not have been peer-reviewed. Any opinions or recommendations discussed are solely those of the author(s) and are not endorsed by BMJ. BMJ disclaims all liability and responsibility arising from any reliance placed on the content. Where the content includes any translated material, BMJ does not warrant the accuracy and reliability of the translations (including but not limited to local regulations, clinical guidelines, terminology, drug names and drug dosages), and is not responsible for any error and/or omissions arising from translation and adaptation or otherwise.

Open access This is an open access article distributed in accordance with the Creative Commons Attribution Non Commercial (CC BY-NC 4.0) license, which permits others to distribute, remix, adapt, build upon this work non-commercially, and license their derivative works on different terms, provided the original work is properly cited, appropriate credit is given, any changes made indicated, and the use is non-commercial. See: http://creativecommons.org/licenses/by-nc/4.0/.

ORCID iD

Yu Xu http://orcid.org/0000-0001-9819-1965

\section{REFERENCES}

1 Carcangiu ML KR, Carcangiu ML, et al. WHO classification of tumours of female reproductive organs. International Agency for Research on Cancer, 2014.

2 Hendrickson M, Ross J, Eifel P, et al. Uterine papillary serous carcinoma: a highly malignant form of endometrial adenocarcinoma. Am J Surg Pathol 1982;6:93-108.

3 Lauchlan SC. Tubal (serous) carcinoma of the endometrium. Arch Pathol Lab Med 1981;105:615-8.

4 Touhami O, Trinh X-B, Gregoire J, et al. Is a more comprehensive surgery necessary in patients with uterine serous carcinoma? Int $J$ Gynecol Cancer 2015;25:1266-70.

5 Colombo N, Creutzberg C, Amant F, et al. ESMO-ESGO-ESTRO consensus conference on endometrial cancer: diagnosis, treatment and follow-up. Ann Oncol 2016;27:16-41.

6 Benito V, Lubrano A, Arencibia O, et al. Pure papillary serous tumors of the endometrium: a clinicopathological analysis of 61 cases from a single institution. Int J Gynecol Cancer 2009;19:1364-9.

7 Rosenberg P, Boeyrd B, Simonsen E. A new aggressive treatment approach to high-grade endometrial cancer of possible benefit to patients with stage I uterine papillary cancer. Gynecol Oncol 1993;48:32-7.

8 Slomovitz BM, Burke TW, Eifel PJ, et al. Uterine papillary serous carcinoma (UPSC): a single institution review of 129 cases. Gynecol Oncol 2003;91:463-9.

9 Nicklin JL, Copeland LJ. Endometrial papillary serous carcinoma: patterns of spread and treatment. Clin Obstet Gynecol 1996;39:686-95.

10 Lachance JA, Everett EN, Greer B, et al. The effect of age on clinical/ pathologic features, surgical morbidity, and outcome in patients with endometrial cancer. Gynecol Oncol 2006;101:470-5.

11 Creasman WT, Kohler MF, Odicino F, et al. Prognosis of papillary serous, clear cell, and grade 3 stage I carcinoma of the endometrium. Gynecol Oncol 2004;95:593-6.

12 Hamilton CA, Cheung MK, Osann K, et al. Uterine papillary serous and clear cell carcinomas predict for poorer survival compared to grade 3 endometrioid corpus cancers. Br J Cancer 2006;94:642-6.

13 Chambers JT, Merino M, Kohorn El. Uterine papillary serous carcinoma. Obstet Gynecol 1987;69:109-13.

14 Goff BA, Kato D, Schmidt RA, et al. Uterine papillary serous carcinoma: patterns of metastatic spread. Gynecol Oncol 1994;54:264-8.

15 Koh W-J, Abu-Rustum NR, Bean S, et al. Uterine neoplasms, version 1.2018, NCCN clinical practice guidelines in oncology. J Natl Compr Canc Netw 2018;16:170-99.

16 Kawaguchi R, Matsumoto K, Akira S, et al. Guidelines for office gynecology in Japan: Japan Society of obstetrics and gynecology (JSOG) and Japan association of obstetricians and Gynecologists (JAOG) 2017 edition. J Obstet Gynaecol Res 2019;45:766-86. 
17 Ruvalcaba-Limón E, Cantú-de-León D, León-Rodríguez E. [The first Mexican consensus of endometrial cancer. Grupo de Investigación en Cáncer de Ovario y Tumores Ginecológicos de México]. Revista de investigacion clinica; organo del Hospital de Enfermedades de la Nutricion 2010;62:583:585-605

18 Lee SW, Lee TS, Hong DG, et al. Practice guidelines for management of uterine corpus cancer in Korea: a Korean Society of gynecologic oncology consensus statement. J Gynecol Oncol 2017;28:e12.

19 Gehrig PA, Groben PA, Fowler WC, et al. Noninvasive papillary serous carcinoma of the endometrium. Obstet Gynecol 2001;97:153-7.

20 Gehrig PA, Van Le L, Fowler WC. The role of omentectomy during the surgical staging of uterine serous carcinoma. Int J Gynecol Cancer 2003;13:212-5.

21 Eitan R, Gershoni A, Sabah G, et al. Surgical staging for uterine papillary serous carcinoma: is omentectomy really necessary? Gynecol Oncol 2013;130:e70.

22 Hui P, Kelly M, O'Malley DM, et al. Minimal uterine serous carcinoma: a clinicopathological study of 40 cases. Mod Pathol 2005;18:75-82.

23 Kaban A, Topuz S, Erdem B, et al. Is omentectomy necessary for non-endometrioid endometrial cancer. Gynecol Obstet Invest 2018;83:482-6.

24 Luz R, MacDonald N, Mould T. Omental biopsy for surgica staging of uterine serous carcinoma. Int J Gynecol Cancer 2016;26:1448-54.

25 Nieto JJ, Gornall R, Toms E, et al. Influence of omental biopsy on adjuvant treatment field in clinical stage I endometrial carcinoma. BJOG 2002:109:576-8.

26 Stroup DF, Berlin JA, Morton SC, et al. Meta-analysis of observational studies in epidemiology: a proposal for reporting. Meta-analysis of observational studies in epidemiology (moose) group. JAMA 2000;283:2008-12.

27 Moher D, Liberati A, Tetzlaff J, et al. Preferred reporting items for systematic reviews and meta-analyses: the PRISMA statement. PLoS Med 2009;6:e1000097.
28 Preferred reporting items for systematic review and meta-analysis protocols (PRISMA-P) 2015: elaboration and explanation. BMJ 2016;354:i4086.

29 Munn Z, Moola S, Riitano D, et al. The development of a critical appraisal tool for use in systematic reviews addressing questions of prevalence. Int J Health Policy Manag 2014;3:123-8.

30 Munn Z, Moola S, Lisy K, et al. Methodological guidance for systematic reviews of observational epidemiological studies reporting prevalence and cumulative incidence data. Int J Evid Based Healthc 2015;13:147-53.

31 Hakulinen T, Dyba T. Precision of incidence predictions based on Poisson distributed observations. Stat Med 1994;13:1513-23.

32 Higgins JPT, Thompson SG, Deeks JJ, et al. Measuring inconsistency in meta-analyses. BMJ 2003;327:557-60.

33 Huedo-Medina TB, Sánchez-Meca J, Marín-Martínez F, et al. Assessing heterogeneity in meta-analysis: Q statistic or 12 index? Psychol Methods 2006;11:193-206.

34 Egger M, Davey Smith G, Schneider M, et al. Bias in meta-analysis detected by a simple, graphical test. BMJ 1997;315:629-34.

35 Boruta DM, Gehrig PA, Groben PA, et al. Uterine serous and grade 3 endometrioid carcinomas: is there a survival difference? Cancer 2004;101:2214-21

36 del Carmen MG, Birrer M, Schorge JO. Uterine papillary serous cancer: a review of the literature. Gynecol Oncol 2012;127:651-61.

37 Brooks RA, Fleming GF, Lastra RR, et al. Current recommendations and recent progress in endometrial cancer. CA Cancer J Clin 2019;69:258-79.

38 Chen M, Guo P, Tan J, et al. The role of omentectomy in the surgical treatment of uterine serous carcinoma. Eur J Obstet Gynecol Reprod Biol X 2019;4:100084.

39 Chan JK, Loizzi V, Youssef M, et al. Significance of comprehensive surgical staging in noninvasive papillary serous carcinoma of the endometrium. Gynecol Oncol 2003;90:181-5.

40 Geisler JP, Geisler HE, Melton ME, et al. What staging surgery should be performed on patients with uterine papillary serous carcinoma? Gynecol Oncol 1999;74:465-7. 